\section{Absence of Bile Salts in the Gastric Juice of a Crab}

THe digestive juices of several decapod Crustacea have been shown to possess a low surface tension and emulsifying properties indicating the presence of surface-autive compounds ${ }^{1}$. Earlier claims ${ }^{2,3}$ of the presence of taurodeoxycholic acid in the gastric juice of the crab and the crayfish have not been supported by recent investigations ${ }^{4}$ which have demonstrated some differences in the properties of the emulsifiers and those of bile salts.

The aim of the work recorded here was to establish by means of chromatographic and mass-spectrometric methods the presence or absence of bile salts in crab (Cancer pagurus L.) gastric juice.

Gastric juice was collected as described by Vonk ${ }^{4}$ from freshly caught crabs and the juice was freeze-dried. The dried juice was extracted with hot ethanol and the alcohol extract was evaporated to dryness under reduced pressure. The residue $(4 \cdot 25 \mathrm{~g}$ corresponding to $85 \mathrm{ml}$. of fresh juice) was hydrolysed with $2 \mathrm{M}$ methanolic potassium hydroxide at reflux temperature for $1 \mathrm{~h}$. The hydrolysis mixture was diluted with water, acidified to $p \mathrm{H} l$ with hydrochloric acid and extracted first with ethyl ether and then with water-saturated $n$-butanol. Both extracts were washed with water until neutral and evaporated to dryness under reduced pressurc. The weight of the residue of the ether oxtract was $150 \mathrm{mg}$ and of the butanol extract $1.9 \mathrm{~g}$. The residue of the ether extract was chromatographed on a $13.5 \mathrm{~g}$ column of hydrophobic 'Hyflo SuperCel' using phase system $F 2$ (ref. 5). Titration of the offluent showed the presence of four peaks with a combined weight of $48 \mathrm{mg}$. After methylation with diazomethano an aliquot of each poak was subjected to gas chromatography using $Q F-1$ as stationary phase and under the conditions described by Sjövall ${ }^{6}$. The main components emerged from the column at much lower temperatures than do bile acid methyl esters and bile sterols. Mass-spectrometry of the material in the four peaks showed that two of these consisted of decanoic acid and $n$-dodecenoic acid respectively, whoreas the material in the other two peaks could not be identified. No frag. ments typical of steroids or bile acids were found. The alcohol eluate of the column $(91 \mathrm{mg})$ was found to consist mainly of cholesterol identified by melting point $\left(147^{\circ}\right.$ $148^{\circ}$ ) with no depression on admixture of authentic cholesterol, and by thin-layer chromatography (benzene/ ethyl acetate, $4: 1$, as moving phase).

The residue of the butanol extract was chromatographed on a 45-g column of hydrophobic 'Hyflo SuperCel' using phase system $D$ (ref. 7). A single peak appearing shortly after the solvent front was obtained. This material $(913 \mathrm{mg})$ was somewhat less polar than taurodeoxycholic acid both on paper and on thin-layer chromatography ${ }^{8,9}$. Neither from the remaindor of the effluent nor from the alcohol eluate of the column could any material be isolated that on gas chromatography or mass-spectrometry showed properties of bile acids or steroids. The infra-red spectrum of the unknown material isolated from the butanol extract as described here showed peaks at 6.0 and $6.5 \mu$, indicating the presence of amide groups. Due to the low volatility no mass-spectrum could be obtained. The unknown material was hydrolysed with 1 M sodium hydroxide in 50 per cent aqueous ethanol for $8 \mathrm{~h}$ in a sealed steol bomb at $120^{\circ}$. After dilution with water and acidification, the hydrolysis mixture was extracted with ether. The residue of the ether extract weighed $359 \mathrm{mg}$. Gas chromatography of an aliquot of the other extract after methylation showed the presence of a number of compounds emerging from the column at temperatures lower than those required for bile acid methyl esters and bile sterols. Direct mass-spectrometric analysis of the gas chromatographic effluent ${ }^{10}$ showed that the material in the ether extract consisted of a series of saturated and unsaturated straight-chain fatty acids with chain-lengths of 10-24 carbon atoms. The water phase of the hydrolysis mixture was passed through a 'Dowex $50-X$ 8' column (hydrogen form; $200 \mathrm{~mm} \times 10 \mathrm{~mm}$ ). The column was washed with water until the effluent was neutral and then with an additional $50 \mathrm{ml}$. of water. The effluent was evaporated to dryness under reducod pressure. Crystallization of the residue from methanol/water yielded 132 mg of taurine identified by thin-layer chromatography (ethanol/ammonia/water, 90:5:5, as moving phase ${ }^{11}$ ) and by formation of $N$-acetyltaurine with m.p. $190^{\circ}$, not depressed on admixture of authentic $N$-acetyltaurine. Thin-layer chromatography of the mother liquor showed that in addition to taurine another compound moving as sarcosine was present. The compound could be isolated by alcohol extraction of the residue of the mother liquor, followed by crystallization from water/acetone/methylene chloride. The crystals had m.p. $168^{\circ}$ with no depression on admixture of authentic sarcosine.

The results of the present investigation show that no bile acids or bile alcohols are present in gastric juice of the crab. However, material possessing emulsifying properties can be isolated from an alcohol extract of dried gastric juice. This material, which has the same properties as that earlier investigated by Vonk, has been shown to consist of fatty acids, taurine and sarcosine.

\section{A. VAN DEN OORD}

Laboratory for Comparative Physiology, Ǔniversity of Utrecht.

Department of Chemistry,

\section{H. Danielsson}

Laboratory for Mass Spectrometry, Karolinska Institutet, Stockholm.

1 Vonk, H. J., Z. vergl. Physiol., 21, 717 (1935).

${ }^{2}$ Vonk, H. J., Bull. Soc. Chim. Biol., 29, 94 (1947).

s Vonk, H. J., in The Physiology of Crustacea, edit. by Waterman, T. H., 1, 291 (Academic Press, New York, 1960).

- Vonk, H. J., Arch. Intern. Physiol. Biochim., 70, 67 (1962).

s Norman, A., and Sjövall, J., J. Biol. Chem., 238, 872 (1958).

- Sjövall, J., Acta Chem. Scand., 16, 1761 (1962).

"Norman, A., Acta Chem. Scand., 7, 1413 (1953).

${ }^{8}$ Sjövall, J., Clin. Chim. Acta, 4, 652 (1959).

- Gänshirt, H., Koss, F. W., and Morianz, K., Arzneimittel-Forsch., 10, 943 (1960).

${ }^{10}$ Ryhage, R., Anal. Chem., 36, 759 (1964).

${ }^{11}$ Strack, E., and Lorenz, J., Z. physiol. Chem., 298, 27 (1954).

\section{Nuclear Magnetic Resonance of Phosphorus in Deoxythymidine Polynucleotides}

NUCLEAR magnetic resonance signals from polynucleotides of thymidine-5' -phosphate have been investigated and the terminal monoester phosphate distinguished from the diester phosphate of the chain.

A mixture of $3^{\prime}$-O-acetylthymidine-5'-phosphate (1 m.mole) and thymidine- $5^{\prime}$-phosphate $(3 \mathrm{~m}$.mole) was polymerized with dicyclohexylcarbodiimide and the resulting mixture of polynucleotides was resolved on a DEAEcellulose (carbonate) column using the method described by Khorana and Vizsolyi ${ }^{1}$. From the polymerization mixture, 26,500 optical density (OD) units at $267 \mathrm{~m} \mu$ ( 1 m.mole of thymidine- $5^{\prime}$-phosphate is equivalent to 9,600 OD units) were applied to the column and polynucleotides up to the dodecanucleotide were cleanly separated. Fractions were identified using paper chromstography with reference samples, prepared in the above manner by Khorana and Vizsolyi and characterized enzymatically by Dr. W. E. Razzell of Syntex Institute. Pooled fractions containing cyclic dinucleotide (3,300 OD units), linear dinucleotide (2,700 OD units), linear trinucleotide $(2,600$ OD units), linear tetranucleotide (2,300 OD units), and linear pentanucleotido (2,000 OD units) were evaporated to dryness and each dissolved in $0.5 \mathrm{ml}$. of methanol for the nuclear magnetic resonance measurements. 